\title{
Effect of Educational Program on Adolescent Girl's Awareness regarding Balanced Diet in the Prevention of Postmenopausal Osteoporosis
}

\author{
Heba Refhat Abo el-Fotouh ${ }^{1}$, Moharam Abd Elhasib Elnafrawy ${ }^{2}$, Soad Abd Elsalam \\ Ramadan $^{3}$ and Amira Mohammed Salama ${ }^{4}$
}

(1)Nursing Specialist at Nursing Secondary School in Kaliop, (2) Professor \& Chairman of Obstetrics and gynecology Department Faculty of Medicine-Banha University, (3)Professor of Obstetrics and Woman Health Nursing Faculty of Nursing -Banha University, and (4) Assist Prof of Obstetrics and Woman Health Nursing Faculty of Nursing-Banha University.

Abstract

Background: Adolescence girls who are known about diet are more likely to avoid osteoporosis at menopause. Osteoporosis is a bone disease in which bone loss occurs, so that bones become weak and more likely to break. The aim of this study was to assess the effect of educational program on adolescent girl's awareness regarding balanced diet in the prevention of postmenopausal osteoporosis. Setting: This study was conducted at secondary nursing school in Benha Teaching Hospital. Two tools were used: I:A structured interviewing questionnaire to assess; a) socio-demographic data of the adolescent girls, b) knowledge of adolescent girl's regarding healthy balanced diet, c) knowledge of adolescent girl's regarding osteoporosis, d ) knowledge of adolescent girl's regarding relationship between un balanced diet and osteoporosis at menopause. II: Likert scale to assess attitude of adolescent girls regarding balanced diet. Results: There was highly statistically significant difference pre and post program implementation regarding total knowledge about balanced diet, total knowledge about osteoporosis and total knowledge about relation between un balanced diet and osteoporosis $(\mathrm{P} \leq 0.001)$ also , there was highly statistically significance correlation between total knowledge score and total attitude score $(\mathrm{P}=$ 0.000) post program implementation while there was no statistical significance correlation preprogram implementation. Conclusion: There was highly statistically significance correlation between total knowledge score and total attitude score $(\mathrm{P}=0.000)$ post program implementation while there was no statistical significance correlation preprogram implementation .Therefore, the study hypothesis was supported. Recommendations: Educational curriculum should include topics of puberty and balanced diet that enable teachers to discuss these subjects with their students and continuous health education program for adolescence must be conducted at school to improve knowledge and attitude of adolescence toward balanced diet.

Key words: Adolescence, balanced diet, knowledge, osteoporosis.

\section{Introduction:}

Adolescence refers to the period of the transition from childhood into adulthood. Historically, it has been defined as being between the ages of 12 and 18 years of age. A recent work by some leading scholars has proposed that an expanded definition and timeframe of 10 to 24 years of age corresponds more closely to adolescent growth and general knowledge of this life period (Fryar et al ., 2019) .

Adolescence can be divided into three stages. Early adolescence (10-13 years) is characterized by a spurt of growth and the beginnings of sexual maturation. Mid- 
adolescence (14-15 years), the main physical changes are completed, while the individual develops a stronger sense of identity. In later adolescence (16-19 years), the body fills out and takes its adult form. Adolescence is the only time following infancy when the rate of physical growth actually increases. For this to happen there is a greater demand for calories and nutrients (Gatachew et al., 2019) .

During adolescence there is a high susceptibility to nutritional deficiencies and poor eating habits. This may lead to problems later on in life such as osteoporosis, obesity, hyperlipedemia, sexual maturation delays, and final adult height (Keats et al ., 2018) . The menopause-related estrogen deprivation is the main cause of osteoporosis, which is characterized by reduction in bone mineral density (BMD) and deterioration of microarchitecture, leading to an increased risk of fractures (Kanis et al ., 2019).

The promotion of healthy habits is very important for reducing the risk of osteoporosis. Bone health is positively influenced by a good general nutritional status and ensuring an adequate dietary intake of calcium, vitamin $\mathrm{D}$ and protein as well as performing regular weight-bearing exercise and avoiding harmful habits, such as alcohol intake and smoking helps to improve bone quality (Williams \& Suchdev, 2017).

Osteoporosis is an asymptomatic bone disease characterized by low bone mass density (BMD) and deterioration of the bone tissues. Bone fragility and susceptibility to fractures, especially hip fractures (PerzaDelgado et al., 2020). The word osteoporosis is derived from the "osteon" which means bone and "porous" which mean little hole. Bone is a very vital organ of the body that not only provides structural support and mobility but it also works as a storehouse of the minerals such as calcium and phosphorous. Bones are made up of minerals and proteins. These proteins and minerals add up various properties to the bones (Brzezinska et al ., 2020).

Osteoporosis is considered a serious problem in developed as well as developing countries. In osteoporosis, bone mass decrease and bone tissue destruction result in an increased risk of fractures. (Rastgoo et al., 2019).

Adolescent girls can prevent occurrence of osteoporosis through promotion of healthy habits that is very important for reducing the risk of osteoporosis. Ensuring an adequate dietary intake of calcium, vitamin $\mathrm{D}$ and protein-as well as performing regular weight-bearing exercise and abandoning harmful habits, such as alcohol intake and smoking-helps to improve bone quality (Leng et al ., 2018).

The nurse assists in health education curriculum development teams and may also provide programs for staff, and families .Community develops strategies to improve healthy eating knowledge, skills, behavior of school students \& provide health information to individual students and groups of students according to their needs (Stacey et al ., 2017)

Significance of the study: Worldwide, it has been estimated that around two billion people have diets inadequate in micronutrients. This, coupled with increasing life expectancies, means that the later years of life are being hampered by partly preventable health issues, including osteoporosis(Kruger \& Wolber, 2016).

\section{Aim of the study:}

To assess effect of educational program on adolescent girls awareness regarding balanced 


\section{Effect of Educational Program on Adolescent Girl's Awareness regarding Relationship between Unbalanced Diet and Osteoporosis at Menopause}

diet in the prevention of postmenopausal osteoporosis.

\section{Research hypothesis:}

Educational program will raise adolescent girl's awareness (knowledge \& Attitude) regarding balanced diet in the prevention of postmenopausal osteoporosis.

\section{Subjects and Method}

\section{Study design:}

Quasi-experimental design was utilized to fulfill the aim of the current study.

\section{Study setting}

This study was conducted at secondary nursing school in Benha Teaching Hospital.

\section{Sample type:}

A purposive sample.

\section{Sample size:}

A total sample of (107) adolescent girls included in the current study who enrolled in the first grade (39), second grade (38), and third grade (30) of secondary nursing school.

\section{Tools of data collection:}

Two tools were used for data collection.

\section{Tool 1: A structured interviewing questionnaire.}

It was designed by researcher based on literature review and written in simple clear Arabic Language. It classified into four parts:

First part : To assess socio-demographic data of the adolescent girls which included 9 items ( age , address, class , School name , Number of family members , father's education level, mother's education level, Father's Job, mother's job ).

Second part : To assess knowledge of adolescent girl's regarding healthy balanced diet which included 19 items ( definition of balanced food, nutritional elements of balanced diet during adolescence, benefits of foods to humans, food pyramid, teenager's nutritional needs, importance of vitamins to human body, number of meals per day, most important nutrient you eat, healthy tips for eating, bad eating habits during adolescence, nutritional errors during adolescence, healthy food you wish eat, calcium rich food , food rich in vitamin $\mathrm{D}$, food rich in protein, food rich in carbohydrates, diet rich in fatty substances, diet rich in mineral salts, reasons that affect calcium absorption in the body .

Third part: To assess knowledge of adolescent girl's regarding osteoporosis. It included 8 items (definition, causes, symptoms, methods for diagnosis, complications, treatment, ways to prevent osteoporosis , factors affect calcium absorption).

Fourth part: To assess knowledge of adolescent girl's regarding relationship between un balanced diet and osteoporosis at menopause. It included 9 items (Incidence, Causes of higher incidence, Malnutrition's definition , element decrease during adolescence leading to osteoporosis at menopause, foods affect calcium absorption , important nutrients for bone health , importance of calcium for body, foods that reduce osteoporosis, foods that increase development of osteoporosis ).

Tool II : Likert scale to assess attitude of adolescent girls regarding balanced diet which was adapted from ( Patimah et al ., 2016),

\section{Tools validity and reliability:}

Content validity of tools was assessed by three of expertise of the Nursing Fields. The expertise reviewed the tool for clarity of sentences, consistency and appropriateness of content, the sequence of items, accuracy, relevance, comprehensiveness, simplicity and applicability of the tools. No modifications 
were done. Reliability of tools was done by cron boch's Alpha Test and it was reliable for knowledge equal 0.73 and reliable for attitude equal 0.71

\section{Ethical considerations:}

- An official permission from the selected study setting was obtained for the fulfillment of the study.

-An oral consent was used to obtain the adolescent girls agreement to participate in the study after explaining the purpose of the study and withdraw- when needed.

- The aim of the study was explained to each adolescent girl before applying the tools to gain their confidence and trust.

-The study didn't have any physical, social or psychological risk on the adolescent girl's.

- The data was collected and treated confidentiality.

\section{Pilot study:}

The pilot study was carried on $10 \%$ of the adolescent girls (11) to test the clarity, practicality and applicability of the study tool as well as the estimation of the time needed to fill the questionnaire. No modifications were done. Adolescent girls involved in the pilot were included in the main study sample.

\section{Field work:}

The researcher visited the pre mentioned setting from 9 a.m to 12 p.m, three days per week (Monday, Tuesday, Thursday ) to collect data from adolescent girls, the study started from the beginning of September, 2020 to the end November , 2020 covering three months. The researcher obtained essential administrative permission from the director of the selected study setting. Data collection procedure was done through four phases; preparatory, assessment, implementation and evaluation phase.

\section{Preparatory Phase:}

It was the first phase of the study and it included reviewing current and related literatures. Also, theoretical knowledge of various aspects of the study using books, articles, periodicals, magazines and internet to develop tools for data collection.

\section{Assessment phase:}

The researcher interviewed the adolescent girls to collect baseline data, at the beginning of interview, greeted them, introduced herself, and explained the aim of the study and took oral consent to participate in the study.

Data was collected by the researcher through the distribution of structured interviewing questionnaire (pretest) to assess adolescent girl's personal data and knowledge regarding balanced diet and osteoporosis and likert scale to assess attitude of adolescent girls regarding balanced diet.

\section{Implementation phase:}

- The educational program was implementing to adolescent girls based on adolescent girls needs after revising tools of data collection at line assessment.

- Researcher started orienting session by providing knowledge regarding balanced diet during adolescence. Then second instruction session concerned with knowledge regarding osteoporosis and third instruction session concerned with relationship between unbalanced diet and osteoporosis at menopause. At the end of session, supportive material (Arabic booklet) was distributed to each adolescent girl.

\section{Evaluation phase:}

The effectiveness of educational program was evaluated by using the same tools

of (pretest) after implementation of educational program ( posttest).

\section{Statistical analysis:}

The collected data were organized, coded, computerized, tabulated and analyzed using appropriate statistical test, the data were analyzed by using the Statistical 


\section{Effect of Educational Program on Adolescent Girl's Awareness regarding Relationship between Unbalanced Diet and Osteoporosis at Menopause}

Package for Social Science (SPSS), that was used to calculate frequencies and percentage mean and standard deviation, also statistical significance and associations by using chisquare test, it is a test used to study association between two qualitative variables and matrix correlation to detect the relation between the variables for ( $\mathrm{P}$ value ) spearman correlation (r).

\section{Results:}

Table (1): Shows that more than one third ( $36.4 \%$ ) of studied students were in age group 15 years old with the mean age $15.90 \pm 0.795$ years . More than three quarters of studied students $(80.4 \%)$ were living in rural areas. Regarding educational class more than one third of studied students $(36.4 \%)$ were in the first class. More than one third $(35.5 \%)$ of studied students had 7 family number, and more than one third of father's and mother's education $(34.6,37.4 \%)$ respectively were university education. The majority ( $98.1 \%$ ) of studied student's father were working while two thirds ( $66.4 \%$ ) of studied student's mother were not working.

Table (2) : Reveals that there was highly statistically significant difference pre and post program implementation regarding total knowledge about balanced diet, total knowledge about osteoporosis and total knowledge about relation between un balanced diet and osteoporosis $(\mathrm{P} \leq 0.001)$

Figure (1): Shows that total knowledge score of studied students improved from (13.1\%) before program implementation to $(46.7 \%)$ after program implementation.

Table (3): Clarifies that; There was highly statistical significant difference regarding all items of knowledge about balanced diet and healthy food wished to eat pre and post program implementation $(\mathrm{p}=<0.001)$ while there was no statistical significant difference regarding number of meals per day pre and post program implementation $(\mathrm{p}=>0.05)$
Figure (2): Clarifies that total positive attitude score improved from (20.6\%) preprogram implementation to $(70.1 \%)$ post program implementation.

Table (4): Revealed that there was statistically significant relation between total knowledge score and student's age , educational class and mother education $(\mathrm{p} \leq$ $0.005)$, and there was no statistically significant relation between total knowledge score and student's residence, father education, father job, mother job $(p>0.005$ ) pre implementation while there was no statistically significant relation between total knowledge score and ( age, residence , educational class, father \&mother education , father \& mother job and family member) post program implementation.

Table (5): Revealed that there was statistically significant relation between total attitude score and students age, educational class ( $p \leq 0.005)$, and there was no statistically significant relation between total attitude score and students

( residence, father \&mother education, father $\&$ mother job and family member $(\mathrm{p}>0.005)$ pre implementation while there was statistically significant relation between total attitude score and student's father education , mother education \& family member ( $\mathrm{p} \leq$ 0.005), and there was no statistical significant relation between total attitude score and students ( age , residence, educational class , father \& mother job) post program implementation.

Table (6): Reveals that there was highly statistically significance correlation between total knowledge score and total attitude score $(\mathrm{P}=0.000)$ post program implementation while there was no statistical significance correlation preprogram implementation. 
Table (1): Distribution of studied students regarding socio demographic characteristics $(\mathbf{n}=107)$

\begin{tabular}{|c|c|c|}
\hline Socio demographic & No & $\%$ \\
\hline \multicolumn{3}{|l|}{ Age } \\
\hline 15 years & 39 & 36.4 \\
\hline 16 years & 38 & 36.3 \\
\hline 17 years & 30 & 27.2 \\
\hline Mean \pm SD & \multicolumn{2}{|c|}{$15.90 \pm 0.795$} \\
\hline \multicolumn{3}{|l|}{ Residence } \\
\hline Rural & 86 & 80.4 \\
\hline Urban & 21 & 19.6 \\
\hline \multicolumn{3}{|l|}{ Educational class } \\
\hline First class & 39 & 36.4 \\
\hline Second class & 38 & 36.3 \\
\hline Third class & 30 & 27.2 \\
\hline \multicolumn{3}{|l|}{ Number of family members } \\
\hline Less than 5 & 21 & 19.6 \\
\hline From 5 to 7 & 48 & 44.1 \\
\hline More than 7 & 38 & 36.3 \\
\hline \multicolumn{3}{|l|}{ Father's education } \\
\hline Illiterate & 21 & 19.6 \\
\hline Reads and writes & 26 & 24.3 \\
\hline Secondary education & 23 & 21.5 \\
\hline University education & 37 & 34.6 \\
\hline \multicolumn{3}{|l|}{ Mother 's education } \\
\hline Illiterate & 20 & 18.7 \\
\hline Reads and writes & 29 & 27.1 \\
\hline Secondary education & 18 & 16.8 \\
\hline University education & 40 & 37.4 \\
\hline \multicolumn{3}{|l|}{ Father's Job } \\
\hline Working & 105 & 98.1 \\
\hline Not working & 2 & 1.9 \\
\hline \multicolumn{3}{|l|}{ Mother's job } \\
\hline Working & 36 & 33.6 \\
\hline Not working & 71 & 66.4 \\
\hline
\end{tabular}




\section{Effect of Educational Program on Adolescent Girl's Awareness regarding Relationship}

between Unbalanced Diet and Osteoporosis at Menopause

Table (2): Distribution of studied students regarding total knowledge score pre and post program implementation ( $\mathbf{n}=\mathbf{1 0 7})$.

\begin{tabular}{|c|c|c|c|c|c|c|}
\hline \multirow{2}{*}{ Items } & \multicolumn{2}{|c|}{ Pre implementation } & \multicolumn{2}{|c|}{ Post implementation } & \multirow[t]{2}{*}{$\mathrm{X}^{2}$} & \multirow[t]{2}{*}{ p-value } \\
\hline & No & $\%$ & No & $\%$ & & \\
\hline \multicolumn{7}{|c|}{ Total knowledge 1:(knowledge about balanced diet) } \\
\hline Good & 14 & 13.1 & 57 & 53.3 & \multirow{3}{*}{41.73} & \multirow{3}{*}{$0.000 * *$} \\
\hline Average & 78 & 72.9 & 47 & 43.9 & & \\
\hline Poor & 15 & 14.0 & 3 & 2.8 & & \\
\hline \multicolumn{7}{|c|}{ Total knowledge 2: (knowledge about osteoporosis ) } \\
\hline Good & 5 & 4.7 & 47 & 43.9 & \multirow{3}{*}{45.64} & \multirow{3}{*}{$0.000 * *$} \\
\hline Average & 95 & 88.8 & 58 & 54.2 & & \\
\hline Poor & 7 & 6.5 & 2 & 1.9 & & \\
\hline \multicolumn{7}{|c|}{ Total knowledge 3 : ( knowledge about relation between un balanced diet and Osteoporosis) } \\
\hline Good & 13 & 12.2 & 56 & 52.4 & \multirow{3}{*}{39.81} & \multirow{3}{*}{$0.000 * *$} \\
\hline Average & 84 & 78.5 & 47 & 43.9 & & \\
\hline Poor & 10 & 9.3 & 4 & 3.7 & & \\
\hline
\end{tabular}

*A statistical significant $\mathbf{p} \leq \mathbf{0 . 0 0 5} *$ A highly statistical significant $\mathrm{p} \leq 0.001$

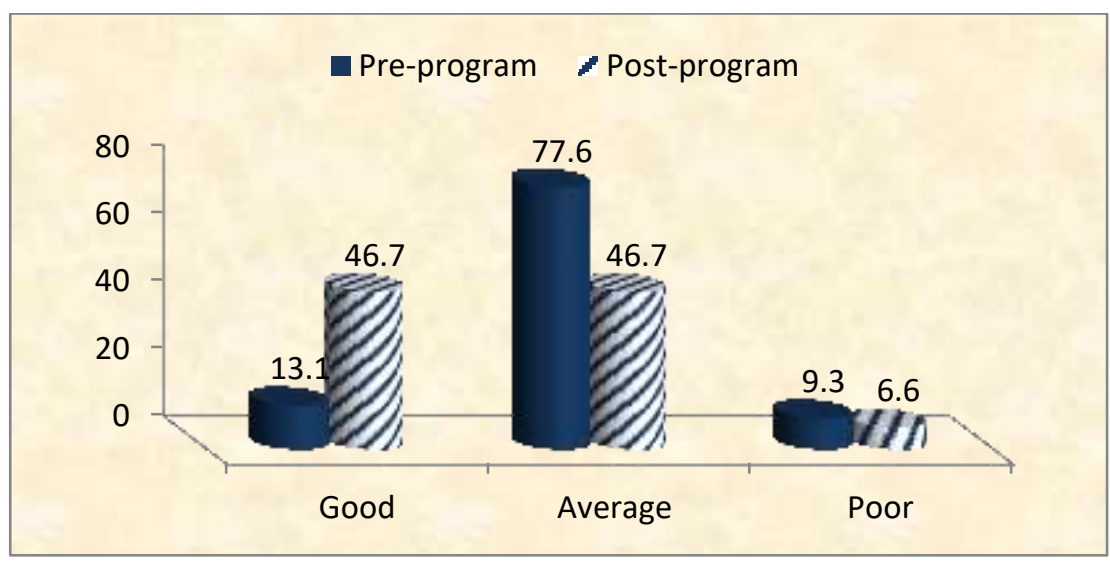

Figure (1): Distribution of studied students regarding total knowledge score $(n=107)$.

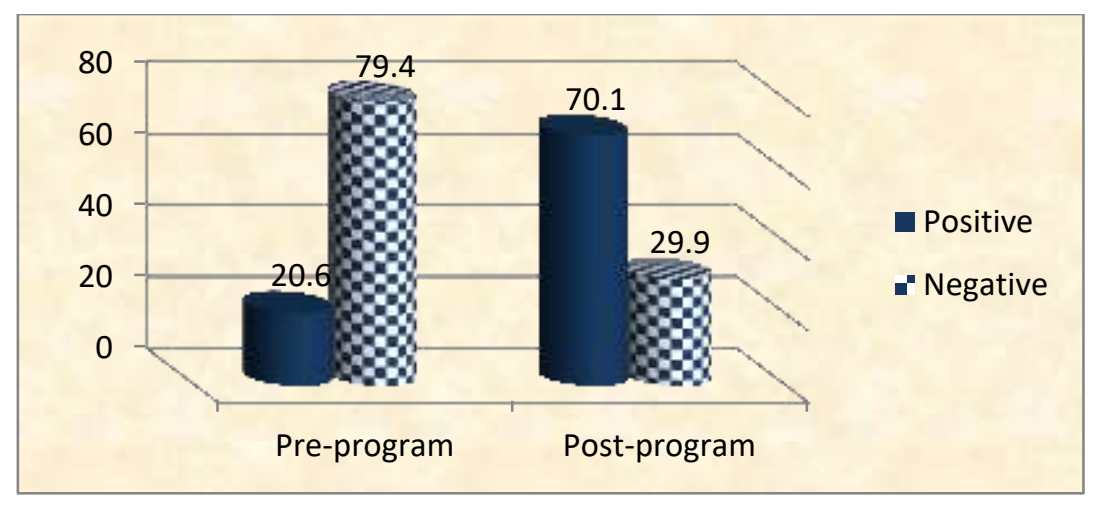

Figure (2): Distribution of studied students regarding their total attitude score $(n=107)$. 
Heba Refhat, Moharam Abd Elhasib, Soad Abd Elsalam and Amira Salama

Table (3): Distribution of studied students regarding knowledge about balanced diet pre and post program implementation $(n=107)$.

\begin{tabular}{|c|c|c|c|c|c|c|c|c|c|c|c|c|c|c|}
\hline \multirow{3}{*}{ Knowledge about balanced diet } & \multicolumn{6}{|c|}{ Pre implementation } & \multicolumn{6}{|c|}{ Post implementation } & \multirow{3}{*}{$\mathbf{X}^{2}$} & \multirow{3}{*}{ p-value } \\
\hline & \multicolumn{2}{|c|}{$\begin{array}{l}\text { Complete } \\
\text { correct } \\
\text { answer }\end{array}$} & \multicolumn{2}{|c|}{$\begin{array}{l}\text { Incomplete } \\
\text { correct } \\
\text { answer }\end{array}$} & \multicolumn{2}{|c|}{ Don't know } & \multicolumn{2}{|c|}{$\begin{array}{c}\text { Complete } \\
\text { correct } \\
\text { answer }\end{array}$} & \multicolumn{2}{|c|}{$\begin{array}{c}\text { Incomplete } \\
\text { correct } \\
\text { answer }\end{array}$} & \multicolumn{2}{|c|}{ Don't know } & & \\
\hline & No & $\%$ & No & $\%$ & No & $\%$ & No & $\%$ & No & $\%$ & No & $\%$ & & \\
\hline Definition of Balanced food & 29 & 27.1 & 78 & 72.9 & - & - & 80 & 74.8 & 27 & 25.2 & - & - & 48.63 & .000 \\
\hline Nutrients during adolescence & 11 & 10.3 & 96 & 89.7 & - & - & 50 & 46.7 & 57 & 53.3 & - & - & 34.87 & .000 \\
\hline Benefits of food to humans & 12 & 11.2 & 93 & 86.9 & 2 & 1.9 & 60 & 56.1 & 47 & 43.9 & - & - & 49.11 & .000 \\
\hline Content of food pyramid's top & 2 & 1.9 & - & - & 105 & 98.1 & 41 & 38.3 & 1 & .9 & 65 & 60.7 & 45.78 & .000 \\
\hline Teenager's nutritional needs & 54 & 50.5 & 1 & .9 & 52 & 48.6 & 86 & 80.4 & 1 & .9 & 20 & 18.7 & 21.53 & .000 \\
\hline Importance of vitamins to human & 19 & 17.8 & 87 & 81.3 & 1 & 9 & 59 & 55.1 & 47 & 43.9 & 1 & .9 & 32.45 & .000 \\
\hline Number of meals/day & 98 & 91.6 & - & - & 9 & 8.4 & 101 & 94.4 & - & - & 6 & 5.6 & .645 & .422 \\
\hline Most nutrient been eaten & 5 & 4.7 & 102 & 95.3 & - & - & 57 & 53.3 & 50 & 46.7 & - & - & 61.40 & .000 \\
\hline Healthy tips for eating habits & 14 & 13.1 & 92 & 86.0 & 1 & .9 & 54 & 50.5 & 52 & 48.6 & 1 & 9 & 34.64 & .000 \\
\hline Bad eating habits in adolescence & 5 & 4.7 & 99 & 92.5 & 3 & 2.8 & 49 & 45.8 & 55 & 51.4 & 3 & 2.8 & 48.42 & .000 \\
\hline Nutritional errors in adolescence & 6 & 5.6 & 100 & 93.5 & 1 & .9 & 48 & 44.9 & 58 & 54.2 & 1 & .9 & 43.83 & .000 \\
\hline Healthy food wish to eat & 8 & 7.5 & 99 & 92.5 & - & - & 18 & 16.8 & 89 & 83.2 & - & - & 4.378 & .036 \\
\hline Calcium rich food & 2 & 1.9 & 105 & 98.1 & - & - & 35 & 32.7 & 72 & 67.3 & - & - & 35.58 & .000 \\
\hline Food rich in vit D & 5 & 4.7 & 92 & 86.0 & 10 & 9.3 & 34 & 31.8 & 67 & 62.6 & 6 & 5.6 & 26.49 & .000 \\
\hline Food rich in protein & 1 & .9 & 105 & 98.1 & 1 & .9 & 1 & .9 & 36 & 33.6 & 70 & 65.4 & 42.10 & .000 \\
\hline Food rich in carbohydrates & 3 & 2.8 & 94 & 87.9 & 10 & 9.3 & 38 & 35.5 & 68 & 63.6 & 1 & .9 & 41.41 & .000 \\
\hline Diet rich in fatty substances & 6 & 5.6 & 101 & 94.4 & - & - & 44 & 41.1 & 63 & 58.9 & - & - & 37.68 & .000 \\
\hline Diet rich in mineral salts & 1 & .9 & 98 & 91.6 & 8 & 7.5 & 40 & 37.4 & 64 & 59.8 & 3 & 2.8 & 46.50 & .000 \\
\hline
\end{tabular}

*A statistical significant $\mathrm{p} \leq \mathbf{0 . 0 5}$

*Ahighly statistical significant $\mathbf{p} \leq \mathbf{0 . 0 0 1}$ 
Effect of Educational Program on Adolescent Girl's Awareness regarding Relationship between Unbalanced Diet and Osteoporosis at Menopause

Table (4): Relation between students' total knowledge score and socio demographics characteristics pre and post program implementation (n= 107).

\begin{tabular}{|c|c|c|c|c|c|c|c|c|c|c|c|c|c|c|c|c|}
\hline \multirow[t]{3}{*}{ Items } & \multicolumn{6}{|c|}{ Pre implementation } & \multirow[t]{3}{*}{$\mathbf{X}^{2}$} & \multirow{3}{*}{$\begin{array}{l}\text { - } \\
\text { value }\end{array}$} & \multicolumn{6}{|c|}{ Post implementation } & \multirow[t]{3}{*}{$\mathbf{X}^{2}$} & \multirow{3}{*}{$\begin{array}{l}\text { p- } \\
\text { value }\end{array}$} \\
\hline & \multicolumn{2}{|c|}{$\begin{array}{l}\text { Poor }(n= \\
10)\end{array}$} & \multicolumn{2}{|c|}{$\begin{array}{l}\text { Average } \\
(n=83)\end{array}$} & \multicolumn{2}{|c|}{$\begin{array}{l}\text { Good }(n= \\
14)\end{array}$} & & & \multicolumn{2}{|c|}{$\begin{array}{l}\text { Poor }(n= \\
7)\end{array}$} & \multicolumn{2}{|c|}{$\begin{array}{l}\text { Average } \\
(n=50)\end{array}$} & \multicolumn{2}{|c|}{$\begin{array}{l}\text { Good } \\
(n=50)\end{array}$} & & \\
\hline & No & $\%$ & No & $\%$ & No & $\%$ & & & No & $\%$ & No & $\%$ & No & $\%$ & & \\
\hline \multicolumn{17}{|l|}{ Age } \\
\hline 15 & 5 & 50.0 & 24 & 28.9 & 10 & 71.4 & \multirow[t]{3}{*}{11.107} & \multirow[t]{3}{*}{$.025 *$} & 4 & 57.1 & 16 & 32.0 & 19 & 38.0 & \multirow[t]{3}{*}{3.573} & \multirow[t]{3}{*}{0.467} \\
\hline 16 & 3 & 30.0 & 35 & 42.2 & 1 & 7.1 & & & 3 & 42.9 & 18 & 36.0 & 18 & 36.0 & & \\
\hline 17 & 2 & 20.0 & 24 & 28.9 & 3 & 21.4 & & & 0 & 0.0 & 16 & 32.0 & 13 & 26.0 & & \\
\hline \multicolumn{17}{|l|}{ Residence } \\
\hline Rural & 9 & 90.0 & 68 & 81.9 & 9 & 64.3 & \multirow[t]{2}{*}{3.012} & \multirow[t]{2}{*}{0.222} & 5 & 71.4 & 41 & 82.0 & 40 & 80.0 & \multirow[t]{2}{*}{0.443} & \multirow[t]{2}{*}{0.801} \\
\hline Urban & 1 & 10.0 & 15 & 18.1 & 5 & 35.7 & & & 2 & 28.6 & 9 & 18.0 & 10 & 20.0 & & \\
\hline \multicolumn{17}{|l|}{ Educational class } \\
\hline First & 5 & 50.0 & 24 & 28.9 & 10 & 71.4 & \multirow[t]{3}{*}{11.107} & \multirow[t]{3}{*}{ 0.025* } & 4 & 57.1 & 16 & 32.0 & 19 & 38.0 & \multirow[t]{3}{*}{3.573} & \multirow[t]{3}{*}{0.467} \\
\hline Second & 3 & 30.0 & 35 & 42.2 & 1 & 7.1 & & & 3 & 42.9 & 18 & 36.0 & 18 & 36.0 & & \\
\hline Third & 2 & 20.0 & 24 & 28.9 & 3 & 21.4 & & & 0 & 0.0 & 16 & 32.0 & 13 & 26.0 & & \\
\hline \multicolumn{17}{|l|}{ Father education } \\
\hline $\begin{array}{l}\text { Read and writes } \\
\text { (Basic education) }\end{array}$ & 4 & 40.0 & 20 & 24.1 & 2 & 14.3 & \multirow[t]{2}{*}{11.57} & \multirow[t]{2}{*}{0.072} & 1 & 14.3 & 15 & 30.0 & 10 & 20.0 & \multirow[t]{2}{*}{3.778} & 0.707 \\
\hline Secondary & 2 & 20.0 & 20 & 24.1 & 1 & 7.1 & & & 2 & 28.6 & 8 & 16.0 & 13 & 26.0 & & \\
\hline
\end{tabular}


Heba Refhat, Moharam Abd Elhasib, Soad Abd Elsalam and Amira Salama

\begin{tabular}{|c|c|c|c|c|c|c|c|c|c|c|c|c|c|c|c|c|}
\hline University & 3 & 30.0 & 24 & 28.9 & 10 & 71.4 & & & 2 & 28.6 & 16 & 32.0 & 19 & 38.0 & & \\
\hline Illiterate & 1 & 10.0 & 19 & 22.9 & 1 & 7.1 & & & 2 & 28.6 & 11 & 22.0 & 8 & 16.0 & & \\
\hline \multicolumn{17}{|l|}{ Mother education } \\
\hline $\begin{array}{l}\text { Read and writes } \\
\text { (Basic education) }\end{array}$ & 2 & 20.0 & 26 & 31.3 & 1 & 7.1 & \multirow[t]{4}{*}{15.665} & \multirow[t]{4}{*}{ 0.016* } & 1 & 14.3 & 15 & 30.0 & 13 & 26.0 & \multirow[t]{4}{*}{6.783} & \multirow[t]{4}{*}{0.341} \\
\hline Secondary & 4 & 40.0 & 13 & 15.7 & 1 & 7.1 & & & 3 & 42.9 & 5 & 10.0 & 10 & 20.0 & & \\
\hline University & 2 & 20.0 & 27 & 32.5 & 11 & 78.6 & & & 2 & 28.6 & 18 & 36.0 & 20 & 40.0 & & \\
\hline Illiterate & 2 & 20.0 & 17 & 20.5 & 1 & 7.1 & & & 1 & 14.3 & 12 & 24.0 & 7 & 14.0 & & \\
\hline \multicolumn{17}{|l|}{ Father job } \\
\hline Working & 10 & 100.0 & 81 & 97.6 & 14 & 100.0 & \multirow[t]{2}{*}{0.589} & \multirow[t]{2}{*}{0.745} & 7 & 100.0 & 49 & 98.0 & 49 & 98.0 & \multirow[t]{2}{*}{0.143} & \multirow[t]{2}{*}{0.931} \\
\hline Not working & 0 & 0.0 & 2 & 2.4 & 0 & 0.0 & & & 0 & 0.0 & 1 & 2.0 & 1 & 2.0 & & \\
\hline \multicolumn{17}{|l|}{ Mother job } \\
\hline Working & 4 & 40.0 & 24 & 28.9 & 8 & 57.1 & \multirow[t]{2}{*}{4.475} & \multirow[t]{2}{*}{0.107} & 2 & 28.6 & 17 & 34.0 & 17 & 34.0 & \multirow[b]{2}{*}{0.086} & \multirow[t]{2}{*}{0.958} \\
\hline Not working & 6 & 60.0 & 59 & 71.1 & 6 & 42.9 & & & 5 & 71.4 & 33 & 66.0 & 33 & 66.0 & & \\
\hline \multicolumn{17}{|l|}{ Family member } \\
\hline Less than 5 & 1 & 10.0 & 12 & 14.5 & 1 & 7.1 & \multirow[t]{3}{*}{3.156} & \multirow[t]{3}{*}{0.532} & 0 & 0.0 & 7 & 14.0 & 7 & 14.0 & \multirow[t]{3}{*}{3.491} & \multirow[t]{3}{*}{0.479} \\
\hline From 5to 7 & 9 & 90.0 & 56 & 67.5 & 10 & 71.4 & & & 7 & 100.0 & 33 & 66.0 & 35 & 70.0 & & \\
\hline More than 7 & 0 & 0.0 & 15 & 18.1 & 3 & 21.4 & & & 0 & 0.0 & 10 & 20.0 & 8 & 16.0 & & \\
\hline
\end{tabular}


Effect of Educational Program on Adolescent Girl's Awareness regarding Relationship between Unbalanced Diet and Osteoporosis at Menopause

Table (5): Relation between students' total attitude score and socio demographic characteristics pre and post program implementation $(n=107)$

\begin{tabular}{|c|c|c|c|c|c|c|c|c|c|c|c|c|}
\hline \multirow{3}{*}{ Items } & \multicolumn{4}{|c|}{ Pre implementation } & \multirow[t]{3}{*}{$\mathbf{X}^{2}$} & \multirow{3}{*}{$\begin{array}{l}\text { p- } \\
\text { value }\end{array}$} & \multicolumn{4}{|c|}{ Post implementation } & \multirow[t]{3}{*}{$X^{2}$} & \multirow{3}{*}{$\begin{array}{l}\text { p- } \\
\text { value }\end{array}$} \\
\hline & \multicolumn{2}{|c|}{$\begin{array}{l}\text { Negative } \\
(n=85)\end{array}$} & \multicolumn{2}{|c|}{$\begin{array}{l}\text { Positive } \\
(n=22)\end{array}$} & & & \multicolumn{2}{|c|}{$\begin{array}{l}\text { Negative } \\
(n=32)\end{array}$} & \multicolumn{2}{|c|}{$\begin{array}{l}\text { Positive } \\
(n=75)\end{array}$} & & \\
\hline & No & $\%$ & No & $\%$ & & & No & $\%$ & No & $\%$ & & \\
\hline \multicolumn{13}{|l|}{ Age } \\
\hline 15 & 24 & 28.2 & 15 & 68.2 & \multirow{3}{*}{12.419} & \multirow{3}{*}{$.002 *$} & 12 & 37.5 & 27 & 36.0 & \multirow{3}{*}{0.085} & \multirow{3}{*}{0.958} \\
\hline 16 & 36 & 42.4 & 3 & 13.6 & & & 11 & 34.4 & 28 & 37.3 & & \\
\hline 17 & 25 & 29.4 & 4 & 18.2 & & & 9 & 28.1 & 20 & 26.7 & & \\
\hline \multicolumn{13}{|l|}{ Residence } \\
\hline Rural & 69 & 81.2 & 17 & 77.3 & \multirow{2}{*}{0.169} & \multirow{2}{*}{0.681} & 23 & 71.9 & 63 & 84.0 & \multirow{2}{*}{2.09} & \multirow[t]{2}{*}{0.148} \\
\hline Urban & 16 & 18.8 & 5 & 22.7 & & & 9 & 28.1 & 12 & 16.0 & & \\
\hline \multicolumn{13}{|c|}{ Educational class } \\
\hline First & 24 & 28.2 & 15 & 68.2 & \multirow{3}{*}{12.419} & \multirow{3}{*}{$.002 *$} & 12 & 37.5 & 27 & 36.0 & \multirow{3}{*}{0.085} & \multirow{3}{*}{0.958} \\
\hline Second & 36 & 42.4 & 3 & 13.6 & & & 11 & 34.4 & 28 & 37.3 & & \\
\hline Third & 25 & 29.4 & 4 & 18.2 & & & 9 & 28.1 & 20 & 26.7 & & \\
\hline Father educatio & & & & & & & & & & & & \\
\hline Read and writes & 20 & 23.5 & 6 & 27.3 & & & 11 & 34.4 & 15 & 20.0 & & \\
\hline Secondary & 20 & 23.5 & 3 & 13.6 & 1782 & 0610 & 11 & 34.4 & 12 & 16.0 & 1084 & $012 *$ \\
\hline University & 30 & 35.3 & 7 & 31.8 & 1.105 & 0.019 & 8 & 25.0 & 29 & 38.7 & 10.004 & .015 \\
\hline Illiterate & 15 & 17.6 & 6 & 27.3 & & & 2 & 6.3 & 19 & 25.3 & & \\
\hline Mother educati & & & & & & & & & & & & \\
\hline Read and writes & 23 & 27.1 & 6 & 27.3 & & & 15 & 46.9 & 14 & 18.7 & & \\
\hline Secondary & 17 & 20.0 & 1 & 4.5 & 3665 & 0300 & 7 & 21.9 & 11 & 14.7 & 12031 & $005 *$ \\
\hline University & 29 & 34.1 & 11 & 50.0 & 0.000 & 0.500 & 8 & 25.0 & 32 & 42.7 & 12.501 & \\
\hline Illiterate & 16 & 18.8 & 4 & 18.2 & & & 2 & 6.3 & 18 & 24.0 & & \\
\hline Father Job & & & & & & & & & & & & \\
\hline Working & 83 & 97.6 & 22 & 100.0 & 0520 & 0460 & 31 & 96.9 & 74 & 98.7 & ( 202 & $0=521$ \\
\hline Not working & 2 & 2.4 & 0 & 0.0 & $0.5 \angle 0$ & 0.400 & 1 & 3.1 & 1 & 1.3 & 0.593 & 0.531 \\
\hline Mother Job & & & & & & & & & & & & \\
\hline Working & 27 & 31.8 & 9 & 40.9 & & 0410 & 8 & 25.0 & 28 & 37.3 & & \\
\hline Not working & 58 & 68.2 & 13 & 59.1 & 0.655 & 0.418 & 24 & 75.0 & 47 & 62.7 & 1.528 & 0.216 \\
\hline Family member & & & & & & & & & & & & \\
\hline Less than 5 & 11 & 12.9 & 3 & 13.6 & & & 1 & 3.1 & 13 & 17.3 & & \\
\hline From 5 to 7 & 59 & 69.4 & 16 & 72.7 & 0.201 & 0.904 & 22 & 68.8 & 53 & 70.7 & 6.939 & $.031 *$ \\
\hline More than 7 & 15 & 17.6 & 3 & 13.6 & & & 9 & 28.1 & 9 & 12.0 & & \\
\hline
\end{tabular}

*A statistical significant $\mathbf{p} \leq \mathbf{0 . 0 0 5}$

*A highly statistical significant $\mathbf{p} \leq \mathbf{0 . 0 0 1}$ 
Table (6): Correlation between total knowledge score and total attitude score among studied students pre and post program implementation

\begin{tabular}{|c||c|c|c|c|}
\hline \multirow{2}{*}{ Item } & \multicolumn{4}{|c|}{ Total knowledge score } \\
\cline { 2 - 5 } & \multicolumn{2}{|c|}{ Pre implementation } & \multicolumn{2}{c|}{ Post implementation } \\
\cline { 2 - 5 } & $\mathbf{R}$ & p-value & $\mathbf{R}$ & p-value \\
\hline \hline Total attitude & 0.134 & 0.169 & 0.69 & $0.000^{* *}$ \\
\hline
\end{tabular}

\section{Discussion:}

Regarding socio- demographic characteristics of the studied students. The present study revealed that, the age of studied sample ranged from 15-17 years old with mean of $15.90 \pm 0.795$. The researcher selected this age to improve knowledge about balanced diet early to avoid osteoporosis at menopause. This results comes in agreement with Fatemeh \&Soheila, (2017) who performed a study on " Effect of educational intervention program for parents on adolescent's nutritional behaviors in Ifahan " and selected the sample in their study from 14-18 years old . But Leila et al .,( 2019) who performed a study on "Life style and preventive behaviors of osteoporosis among women of reproductive age in Qazvin Iran, " selected the sample 's age between 26-35 years old with mean of $30.75 \pm 7.47$ years old.

Concerning residence, the finding of the current study indicated that more than three quarters of studied students were living in rural areas. This result disagrees with Bland et al., (2020) who performed a study on "Physical activity, sedentary time, and longitudinal bone strength in adolescent girls. Tucson "and reported that the highest percentage of the student's girl's residence is urban.

Education is considered as one of the decisive and highly influential factor in reproductive behavior. The present study showed that regarding parent's education, more than one third of father's and mother's education were university education .This result in agreement with Ghobad et al ., (2020) who performed a study on " The prevalence of physical inactivity in Iranian adolescents and the impact of economic and social inequalities on it in Iran "and mentioned that parents of most respondents has university education. This was contrary to Fazeh, (2019) who performed a study on " Osteoporosis preventive behaviors in adolescent girls : The educational program based on BASNEF model in Iran" and mentioned that most of the student's girls and fathers had secondary school education.

Concerning the studied student's knowledge about healthy nutritional habits for protecting bone, the finding of the current study showed that more than half of the studied students had poor knowledge about nutrition related to interested topic. The finding revealed that regarding essential nutritional elements for healthy bone, importance of calcium, and food rich with calcium and food sources of vitamin D. Most studied students had incomplete correct answer about healthy nutritional habits for protecting bone. This may be due to shortage of information and educational programs regarding healthy dietary habits and importance of vitamin D.

These finding are supported by Nasizadeh et al., (2015), who found that 


\section{Effect of Educational Program on Adolescent Girl's Awareness regarding Relationship between Unbalanced Diet and Osteoporosis at Menopause}

majority of students had poor awareness about source of calcium and half of the studied sample, have awareness of calcium intake. But the finding of the current study disagree with Fahd et al ,, (2015) who performed a study on " Knowledge, attitude and practice of osteoporosis among Saudis" and found that the majority of participant had heard about osteoporosis and factors affecting it.

Regarding importance of diet during adolescence period to prevent osteoporosis at menopause. The current study finding indicated that studied students had total poor knowledge about relationship between un balanced diet and osteoporosis pre implementation which improved post implementation as majority of studied students knew that diet was important during adolescence period to avoid osteoporosis at menopause .In the researcher point of view, this may be due to young women don't perceive themselves to be susceptible to osteoporosis; they are not concerned to get information regarding this disease. Also, it may be due to the lack of public awareness campaigns or unavailability of frequent courses about osteoporosis and its preventive measures to promote women's health.. The current study finding is concurrent with AlHemyari et al., (2018) who performed a study on " Assessment of knowledge, attitude and practice of osteoporosis and its predictors among university female students in United Arab Emirates" who mentioned that study aimed to evaluate the knowledge, attitude and practice of university students towards osteoporosis and found that, most of their study participants had previous knowledge about osteoporosis and mentioned that most of students had knowledge that healthy food during this period was important to avoid this disease.
As regard attitude toward nutritional habit, the present study revealed that minority of studied students agree healthy food is not expensive pre intervention and increased to more than a half of studied students agrees with think health food is not expensive after intervention, this may be due to cultural foods $\&$ traditions and educational program improve awareness of studied students about healthy diet component as healthy food not be necessary to be expensive. This finding disagree with Michelle et al ., (2020), who performed a study on " Perceived barriers / facilitators to a healthy lifestyle among diverse adolescents with overweight / obesity" and reported that a majority of the study participants reported that one major barrier to eating healthy meals was the high cost of healthy foods. This may be due to wrong thought about healthy food and may be foods comes from parents and family members who are typically responsible for the foods brought into the home.

Regarding going on healthy diet, the present study finding indicated that most of participant's students continued on doing healthy diet habits as eating vegetables between meals, eating fruits before meals and drinking appropriate amount of water. The researcher explained that more than half of participant's sample preferred to drink appropriate amount of water. This result comes in agreement with Ghada et al ., (2017) who performed a study on " Effect of Health Belief Model Based Nutrition Education on Dietary Habits of Secondary School Adolescent Girls in Sharkia Governorate. Department of Community Medicine, Faculty of Medicine, Zagazig university, Egypt". Who mentioned that highest of percentage of girls followed the healthy food groups and avoid consumption of unhealthy foods (fast, fried, canned foods), 
unhealthy drinks (carbonated and caffeinated drinks), and excess intake of salt and sugar/sweet. While the finding of the present study comes in contrast with Tagreed \& Muhammad , (2020) who performed a study on " Osteoporosis Knowledge, Beliefs and Self- efficacy Among Female University Students" who reported that a few of girls would change to healthy habits that may be related to socio cultural norms and beliefs.

Regarding drinking milk daily, the present study finding revealed that, most of participant's students drink milk daily. This finding comes in agreement with Shi et al ., (2020) who performed a study on " Effect of dairy products on bone mineral density in healthy postmenopausal women " who reported that highest percentage of participants would like to drink milk daily . He explained that women who drink low amount of milk during childhood and adolescence have less bone mass in adulthood and greater risk of fracture and increased milk consumption significantly enhances bone mineral acquisition in adolescent girls and could modify attainment of peak bone mass. But the present study finding comes in contrast with Chamova et al ., (2020) who performed a study on " Nutrition as a bone health factor with significant influence " who reported that a few of girls drink milk daily and preferred eating vegetables as a source of vitamin D and calcium.

Regarding foods affecting absorption of calcium, the present study reported that most of participant's students were informed about foods affecting absorption of calcium which are spinach, beans and sweet. Potatoes that contain oxalic acid that is a potent inhibitor of calcium absorption, citrus that contain (Na) which is major determinant of urinary calcium excretion, and wheat, barely that contain (Affatic acid) which is the most inhibitor of calcium absorption. This study comes in agreement with Somyieh \& Mojgan , (2020) who performed a study on " Effect of education on preventive behaviors of osteoporosis in adolescents " who reported that knowledge about foods affect calcium absorption for prevention of osteoporosis . While this finding comes in contrast with Yun et al ., (2020) who performed a study on " Association between cola consumption and bone mineral density in Korean adolescents and young adults " who reported that few of participants had information about what foods affect calcium absorption.

The findings of the present study revealed that there was statistically significant relation between total knowledge score and student's age, educational class and mother education $(p \leq 0.005)$, and there was no statistically significant relation between total knowledge score and student's residence, father education, father job, mother job ( $\mathrm{p}$ > 0.005 ) pre implementation while there was no statistically significant relation between total knowledge score and ( age, residence, educational class, father \&mother education , father \& mother job and family member) post program implementation

The present study findings displayed that there was statistically significant relation between total knowledge score of studied students and mother's educational level pre implementation while there was no statistical significant relation between total knowledge score of studied students and mother's educational level. Nutritional knowledge was related to dietary intake, highlighting the fact that nutrition - related education and information for mothers can improve their offspring dietary intake. This finding is agree with Walaa , (2018) who performed a study 


\section{Effect of Educational Program on Adolescent Girl's Awareness regarding Relationship between Unbalanced Diet and Osteoporosis at Menopause}

on " Mother's awareness regarding vitamin D deficiency among their infants in kalyobia governorate " and reported that there was a highly statistically significant relation between total knowledge score of mothers and their age, educational level, occupation, monthly income, and residence .

Regarding relationship between total attitude score of studied adolescence and their socio demographic characteristics, the finding of present study revealed that there was statistically significant relation between total attitude score toward healthy nutritional requirements and parent's education. This may be due to that a highly educated parents paid more attention to health and had more opportunities to obtain relevant information and thereby increase knowledge. This results disagree with Kazi et al ., (2018) who performed a study on " Relationships between parent's academic backgrounds and incomes and building students' healthy eating habits " and found that the students have a good knowledge of types of healthy food but yet their preferences are towards the unhealthy food. Though the students' gender and parents' educations are not found significantly related to students' knowledge, attitude and practices towards healthy eating habits, parents' incomes have significant influence on promoting the healthy eating habit. Discussion Findings of this study can be useful to guide parents in healthy food choices.

Regarding the relation between total attitude score and demographic characteristics of studied students, the present study revealed that there was statistically significant relation between total attitude score and students age, educational class ( $\mathrm{p} \leq$ $0.005)$, and there was no statistically significant relation between total attitude score and students ( residence, father \&mother education, father \& mother job and family member ( $p>0.005)$ pre implementation while there was statistically significant relation between total attitude score and student's father education, mother education \& family member ( $\mathrm{p} \leq 0.005)$, and there was no statistical significant relation between total attitude score and students ( age , residence, educational class, father \& mother job ) post program implementation.

The present study revealed that there was statistical significant relation between total attitude score toward healthy nutritional requirements and educational class \& age pre implementation while there was no statistical significant relation between total attitude score and educational class \& age post implementation. This results comes in agreement with Rina et al ., (2019) who reported that there was statistical significant relation between attitude levels and (age \& educational class )as knowledge and attitude levels were significantly higher among females and individuals who were less than thirty years of age. Likewise, knowledge and positive attitude levels were higher among graduates compared to lower education groups.

Additionally, the present study findings represents that there was no statistical significant relation between total attitude score toward healthy nutritional requirements and residence pre and post program implementation, this may be due to change of attitude of studied students was nearly similar in both urban and rural areas. This results comes disagree with Permani et al ., (2020) who performed a study on " Food and nutrition related knowledge, attitude, and practice among reproductive age women in marginalized areas in Sri Lanka" and found that there was statistical significant relation 
between total attitude score about nutritional status and residence as the nutritional status of rural women was significantly better than that of urban women.

Regarding correlation between total knowledge score and total attitude score among studied students in pre and post program implementation, the present study showed that there was highly statistically significance correlation between total knowledge score and total attitude score $(\mathrm{P}=$ 0.000) post program implementation. This result may be due to that educational intervention has a good effect on participant's girls that it improved their knowledge and practices related balanced food. This study comes in agreement with Barzanji et al ., (2016) who performed a study on " Osteoporosis; a study of knowledge, attitude and practice among adults in Riyadh, Saudi Arabia" and found that there was significant correlation between knowledge and attitude regarding osteoporosis. This was probably because the education programs related to health that have been implemented in Saudi Arabia were interesting and could motivate them to have good attitude regarding osteoporosis. This could also be due to the participants who realized that osteoporosis was more prevalent among women.

On the other hand this study disagree with Nabilah et al ., (2018) who performed a study on " Knowledge, attitude and practice regarding osteoporosis among allied health sciences students in a public university in Malaysia " and found that there was no significant correlation between knowledge with attitude and practice regarding osteoporosis. It was perceived that the participants should have better knowledge and positive attitude prior to entry to school, which was a field of medicine that aims to improve health. In addition, it could be because the participants did not pay much attention toward osteoporosis.

From the researcher point of view, educational intervention has a good effect on participant's girls that it improved their knowledge and practices related balanced food. The present study is supported by other studies done by Bial et al ., (2017) who performed a study on " Knowledge, beliefs and practice regarding osteoporosis among female medical students in Pakistan " and Gul \& Tevfik , (2020) who performed a study on " The impact of Health Belief Model Based Educational Intervention on Women's knowledge ,Beliefs Preventive behaviors and clinical outcomes about osteoporosis " they reported that an appropriate diet, moderate exercise , consumption of calcium \& vitamin $\mathrm{D}$ and following healthy lifestyle would prevent occurrence of osteoporosis .

\section{Conclusion:}

There was highly statistical significant difference regarding all items of knowledge about balanced diet, osteoporosis, and relationship between balanced diet and osteoporosis pre and post program implementation. Also, there was highly statistical significant difference pre and post program implementation regarding all items of attitude. Additionally there was highly statistically significance correlation between total knowledge score and total attitude score $(\mathrm{P}=0.000)$ post program implementation while there was no statistical significance correlation preprogram implementation .Therefore, the study hypothesis was supported.

\section{Recommendations:}

- Educational curriculum should include topics of balanced diet during adolescence that enable teachers to discuss these subjects with students and continuous health 
education program for adolescence must be conducted at school to improve knowledge and attitude of adolescence toward balanced diet.

- Use a booklet and posters as methods to increase student's awareness regarding balanced diet and osteoporosis.

- Encourage healthy and positive attitude of young females about healthy balanced diet in early age rather than teaching them when grown up.

- Implementing awareness program about risk factors and preventive measures of osteoporosis.

\section{References:}

Al-Hemyari , s , Jairun AA , Jairoun MA , Metwali $Z$, Maymoun $N$ (2018). Assessment of knowledge , attitude and practice of osteoporosis and its predictors among university students . Journal of advanced pharmacy Education \&Research 8 (3): $43-48$.

Barzanji AT, Alamri FA, Mohamed AG. ( 2016). Osteoporosis: A study of knowledge, attitude and practice among adults in Riyadh, Saudi Arabia. J Community Health 2016; 38(6): 1098-105.

Bial , M ., Hasswb ,A., Merchant, A.Z . et al ., (2017) . Knowledge, beliefs and practice regarding osteoporosis among female medical students in Pakistan. Asia Pac Fam Med 16 , 6 (2017).

Bland V.L , Bea . J.w , Roe D. L et al ., (2020). Physical activity, sedentary time, and longitudinal bone strength in adolescent girls . Osteoporosis International (2020) 31, p: 1943- 1954.

Brzezinska O , Lukasikz, Makowaska J , etal .(2020). Role of vitamin $C$ in osteoporosis development and treatment. Aliterature review .Nutrients 2 (8) : 2394.

Chamova $\mathbf{R}$, Panteleeva $\mathbf{M}$, Ivanova $\mathbf{E}$ (2020). Nutrition as a bone health factor with significant influence . Journal of IMAP 2020; 26 (4), P: 3426- 3431.

Fahd A .Alamri , Mohammed Y . Saeedi, Ashry Mohamed et al ., (2015) : Knowledge , attitude, and practice of osteoporosis among Saudis. Journal of the Egyptian Public Health , Ghobad Moradi , Farideh Mostafavi, Bakhtiar piroozi (2020). The prevalence of physical inactivity in Iranian adolescents and the impact of economic and social inequalities on it :results of a National study in 2018 .BMC public Health 20 , 14-99( 2020).

Fatemeh Mokhtari and Soheila Ehsanpour (2017): Effect of educational intervention program for parents on adolescent's nutritional behaviours in Ifahan. Journal of Education and Health promotion. 2017; 6: 103 .

Fazeh Rastgoo, Parvaneh vasli , Camelia Rohani, et al ., ( 2019). Osteoprosis Preventive behaviors in adolescent girls : The educational program based on BASNEF model . Arch Osteoporosis 14 (71).

Fryar, CD, Carroll, MD, Ogden, CL. (2019). Prevalence of Overweight, Obesity, and Severe Obesity among Children and Adolescents Aged 2-19 Years: United States Hyattsville, MA: National Center for Health Statistics; 2018. https://stacks.cdc.gov/view/cdc/58669.

Accessed May 21, 2019.

Gatachew Arage, Mekonnen Assefa, Teshagerworku (2019). Socio-demographic and economic factors are associated with nutritional status of adolescent school girls . SAGE open Medicine, vol 7 ,No 7. 
Ghada , M ., Salem , Randa , M., et al ., (2017). Effect of Health Belief Model Based Nutrition Education on Dietary Habits of Secondary School Adolescent Girls in Sharkia Governorate . Department of Community Medicine, Faculty of Medicine , Zagazig University.

Gul Pinar ,Tevfik Pinar (2020). " The impact of Health Belief Model Based Educational Intervention on Women's knowledge ,Beliefs Preventive Behaviors and Clinical Outcomes about Osteoporosis . Health Communication, Sociology of Health and illness . Vol 10(3), $90: 171-177$.

Kanis JA, Cooper C, Rizzoli R et al (2019). European guidance for the diagnosis and management of osteoporosis in postmenopausal women. Osteoporos Int $30: 3-4$

Kazi ,E. H ., Kazi ,F. H. , Revethly, T .,( 2018).Relationships between parent's academic backgrounds and incomes and building students' healthy eating habits. Peer J 6 (1) : e 4563.

Keats EC, Rappaport AI, Jain R, Oh C, Shah S, Bhutta ZA.(2018).Diet and eating practices among adolescent girls in low- and middle-income countries: a systemic review. Arlington, VA: Strengthening Partnerships, Results, and Innovations in Nutrition Globally (SPRING) Project; 2018.

Kruger, M., and Wolber, $F$., (2016). Osteoporosis: modern paradigms for last century's bones. Nutrients , 8(6):376

Leila Dehghankar, Mohaddeseh Alakbari, Mafi ( 2019). Life style and preventive behaviors of osteoporosis among women of reproductive age in Qazvin- Iran : A cross sectional study . Social Health and Behavior: 2 (2) , P: 70-75.
Leng LS, Ali A, Yusof HM. (2018). Knowledge, Attitude and Practices towards Osteoporosis prevention among adults in Kuala Lumpur, Malaysia. Malays J Nutr 2018; 23(2): 279-90.

Michelle I , Sarah M , Jackson et al ., (2020). Perceived barriers / facilitators to a healthy lifestyle among diverse adolescents with overweight / obesity, obesity science and practice, available at wiley online library.

Nabilah R , Nor Alzina A, Mainul Haque . (2018). Knowledge, attitude and practice regarding osteoporosis among allied health sciences students in a public university in Malaysia . Erciyes Med J 2018 ; 40 (4) : 210217.

Nasizadeh, M ., Hafezi , A., Bakhtiari , S . M and Harooni , J .,(2015). Osteoporosis related knowledge among students of a medical sciences university in Iran : Calcium intake and physical activity, Journal of Medicine and Life , 8 (4) :203-208.

Nutrition Reports 2017;6(3):228-46.

Patimah., Royani, I., Murslaha , A., and Taha., A.(2016). Knowledge, attitude and practices of balanced diet and correlation with hypochromic microcytic anemia among adolescent school girls in maro District, south Sulawesi,Indonesia-Biomedical Research, 271,pp.165-171.

Permani C , Chandan R, Angelika Ploeger (2020). Food and nutrition related knowledge , attitude, and practice among reproductive age women in marginalized areas in Sri Lanka .International journal of environmental research and public health. (ISSN 16604601).

Perza-Delgado A, Sanchez-GomezMB , Gomez-salgadoJ ,et al (2020). Non- 


\section{Effect of Educational Program on Adolescent Girl's Awareness regarding Relationship}

\section{between Unbalanced Diet and Osteoporosis at Menopause}

pharmacological interventions towards preventing the triad osteoporosis .fall risk-hip fracture, in population older than 65 . Scoping review. Journal of Clinical Medicine 9 (8). 23-29.

Rastgoo F, Vasli P, Rohani C, Amini A (2019). Predictors of osteoporosis preventive behaviors among adolescent. Biomed Res. 2019;30:280-7.

Rina Tripathi , Hafiz A, Makeen, et al ., (2019). Knowledge, attitude and practice about osteoporosis in south - western Saudi Arabia : Across - sectional survey , International Journal of Health Promotion and Education , 57 (1).

Shi, Y., Zhan, Y., Chen, Y. et al (2020). Effect of dairy products on bone mineral density in healthy postmenopausal women: A systematic review and Meta - analysis of randomized controlled trials - Arch osteoporosis 15,48 (2020).

\section{Somyieh Abdolallipour , Mojgan Mirghafour vand (2020). Effect of Education on preventive Behaviors of osteoporosis in Adolescents : A systematic Review and Meta - Analysis . International Quarterly of Community Health Education. Vol $41(2)$.}

\section{Stacey F, Finch M, Wolfenden L, Grady A,} Jessop $\mathrm{K}$, Wedesweiler $\mathrm{T}$, et al (2017).Evidence of the potential effectiveness of centre-based childcare policies and practices on child diet and physical activity: consolidating evidence from systematic reviews of intervention trials and observational studies. Current Williams A., Suchdev P. (2017). Assessing and Improving Childhood Nutrition and Growth Globally. Pediatr Clin N Am 2017;64:755-768 http://dx.doi.org/10.1016/j. pcl.2017.03.001.
Tagreed , O ., Shawashi , Muhammad Darawad (2020) . Osteoporosis Knowledge , Beliefs and Self - efficacy Among Female University Students . The Open Nursing Journal. Department of school of Nursing, The University of Jordan, Amman, Jordan.

Walaa , k., (2018). Mother's awareness regarding vitamin D deficiency among their infants in kalyobia governorate. Community health, available on research gate .August 2018.

Yun A, Kim , Jun, Hyun Yoo (2020). Associations between cola consumption and bone mineral density in Korean adolescents and young adults : across- sectional study using data from the Korea National Health. Journal of Nutritional Science, London .Vol 9 
تأثير برنامج تعليمي علي توعية الفتيات المراهقات عن النظام الغذائي المتوازن في الوقاية من هشاشة العظام بعد سن اليأس الس

هبة رفعت أبو الفتوح - محرم عبد الحسيب النفراوي - سعاد عبد السلام رمضان_أميرة محم سلامة

تعد المر اهقة فترة من النمو السريع و التطورحيث إن اتباع نظام غذائي صحي يمكن أن يساعد في الوقاية من الأمر اض غير المعدية مثل هشاشة العظام ـ لذلك هدفت هذه الدراسة إلي تقييم تأثثر البرنامج التعليمي على توعية الفتيات المر اهقات عن النظام الغذائي المتوازن في الوقاية من هثاشة العظام بعد سن اليأس .وقد أجريت الدر اسة في مدرسة التمريض الثانوية بمسنشفى بنها التعليمي. وأشتشلت على (V • ( ) من الفتيات المراهقات بمدرسة التمريض الثانوية. حيث كثفت النتائج عن أن هناك فروق ذات دلالة إحصائية عالية فيما يتعلق بجميع درجات المعرفة حول النظام الغذائي المتوازن وهشاثـة العظام والعلاقة بين النظام الغذائي المتوازن وهثاشة العظام قبل وبعد تنفيذ البرنامج. أيضا ، كان هناك فروق ذات دلالة إحصائية عالية قبل تنفيذ البرنامج وبعده فيما يتعلق بجميع اتجاهات الطلاب المدروسين فيما يتعلق بالاحتياجات الغذائية الصحية. بالإضافة إلى ذلك ، كان هنالك ارتباط ذو دلالة إحصائية عالية بين مجموع درجات المعرفة ودرجة الاتجاهات بعد تنفيذ البرنامج بينما لم يكن هنالك ارتباط ذات دلالة إحصائية ما قبل تنفيذ البرنامج ، لذلك تم دعم فرضية الدر اسة. كما أوصت الدراسة بأن تتضمن المناهج التعليمية موضوعات النظام الغذائي المتوازن خلال فترة المر اهقة التي تمكن المعلمين من مناقثة هذه الموضوعات مع الطلاب ويجب إجراء برنامج التثقيف الصحي المستمر للمر اهقين في المدرسة لتحسين المعرفة و السلوك للمر اهقين تجاه نظام غذائي متوازن. 